\title{
Characterization of Scedosporium boydii antigens recognized by serum IgGs from cystic fibrosis patients
}

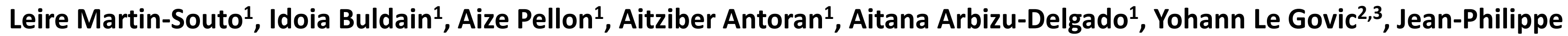
Bouchara $^{2,3}$, Aitor Rementeria ${ }^{1}$, Fernando L. Hernando ${ }^{1}$ \& Andoni Ramirez-Garcia ${ }^{1}$

${ }^{1}$ University of the Basque Country (UPV/EHU), Fungal and Bacterial Biomics Research Group. Dept. of Immunology, Microbiology \& Parasitology, Leioa, Spain

${ }^{2}$ Groupe d'Etude des Interactions Hôte-Pathogène (EA 3142), GEIHP, UNIV. Angers, Université Bretagne-Loire, Angers, France

${ }^{3}$ Laboratoire de Parasitologie - Mycologie, Centre Hospitalier Universitaire d'Angers, Angers, France

\section{INTRODUCTION}

Species of the genus Scedosporium are emergent pathogens ranking the second, only behind Aspergillus spp., among filamentous fungi causing a chronic colonization of the airways of cystic fibrosis (CF) patients. This may lead to chronic inflammation, true respiratory infections like bronchitis, allergic broncho-pulmonary mycoses and fungus ball formations, or even to lifethreatening invasive disease following lung transplantation and in cases of severe immunosuppression.

The lack of standardization and the low sensitivity and specificity of the detection methods commonly used result in a delayed diagnosis. Moreover, these poor detection methods also result in an inadequate monitoring and treatment of the patients. In this sense, species identification is essential for the establishment of an appropriate treatment due to species-specific differences in antifungal susceptibility.

\section{Classification of CF patients' sera}

\section{OBJECTIVE}

To contribute to the finding of new diagnostic targets by identifying the most immunoreactive antigens of Scedosporium boydii recognized specifically by serum IgGs from CF patients colonized by Scedosporium spp.

\section{RESULTS}

CF colonized by Scedosporium spp. (Scedo+)

$$
\text { x5 sera }
$$

$\oplus$ CF colonized by Aspergillus spp. (Asp+)

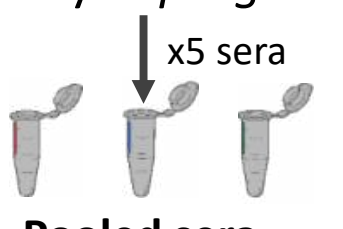

Pooled sera

Control - CF without fungal colonization (Ctrl)

\section{Serological response against S. boydii and A. fumigatus}

Sera from CF patients colonized by Scedosporium spp. showed a greater specific immune response. To compare the serological response against $S$. boydii (A) and A. fumigatus (B), an ELISA assay was performed with serial dilutions of the pooled sera against $50 \mathrm{\mu g} / \mathrm{ml}$ of total protein extract of each fungi (growing during $24 \mathrm{~h}$ in potato dextrose broth at $37^{\circ} \mathrm{C}$ and disrupted with glass beads in a BeadBeater homogenizer), to calculate the titer of IgG antibodies against both fungal species. Regarding CF patients sera colonized by Scedosporium, IgG antibody titer against both fungi was higher (1/2000) than in those with Aspergillus (1/500).

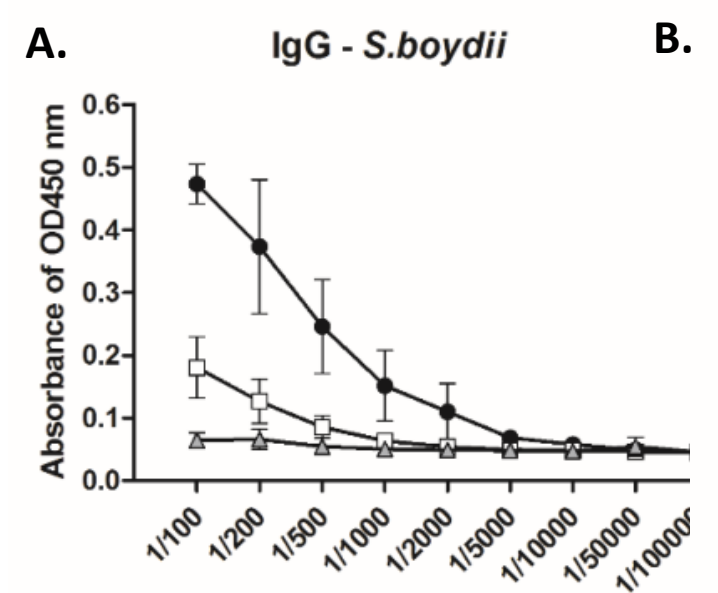

Serial dilutions

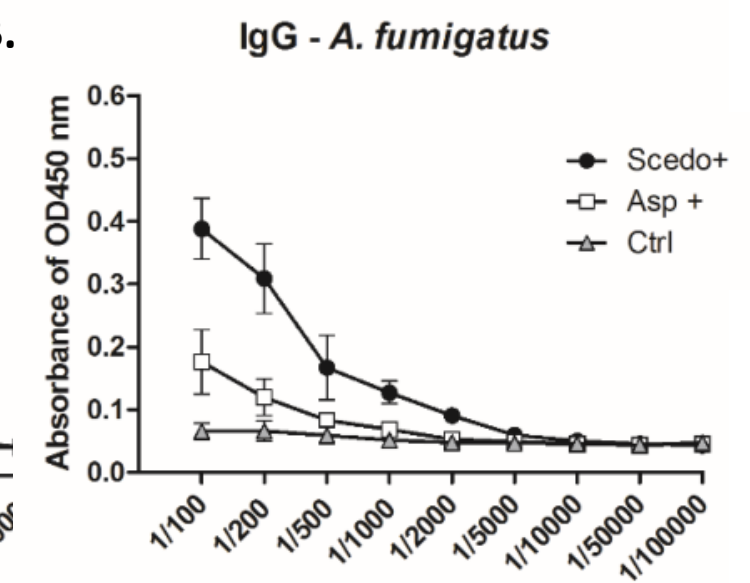

Serial dilutions

\section{Characterization of specific S. boydii antigens recognized by sera from CF patients with Scedosporium}

A.

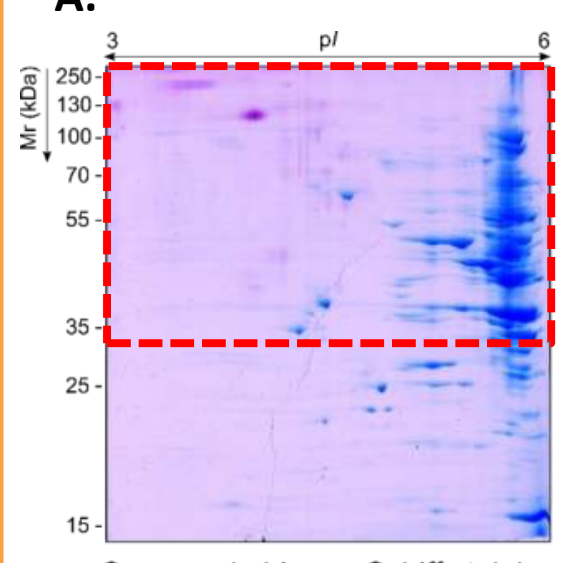

Coomassie blue + Schiff staining

c.

卧,

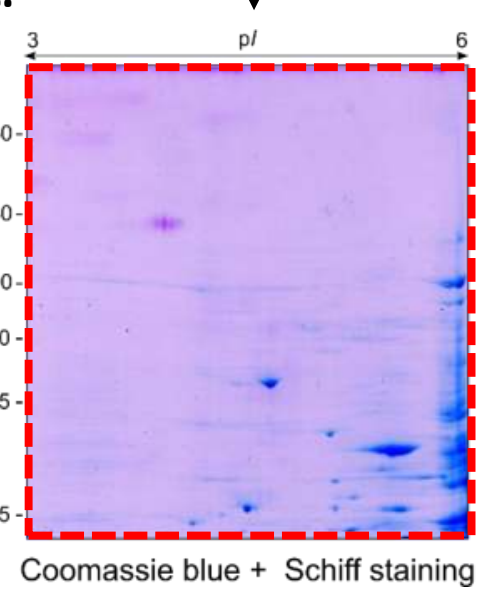

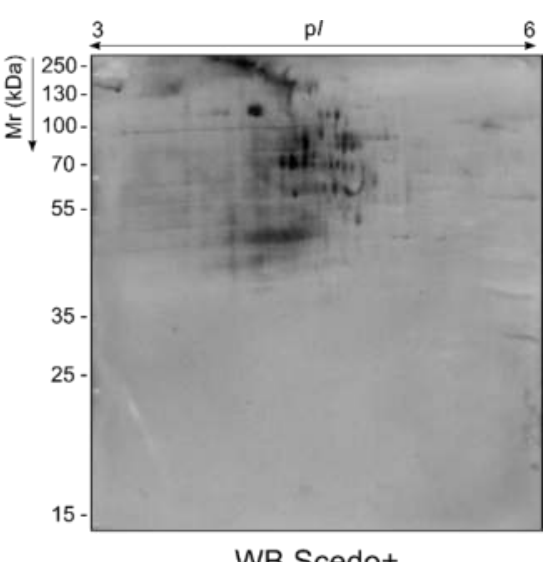

D.

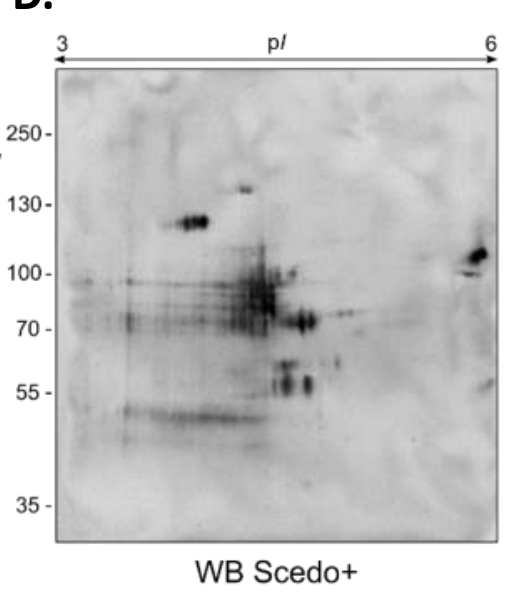

IgG serum antibodies of CF patients with Scedosporium recognized mainly glycoproteins, between 3-5 pl and 45-250 kDa. S. boydii proteome in a 10\% acrylamide gel stained with Coomassie blue and Schiff reagent to visualize proteins (blue) and glycoproteins (purple) respectively (A). Western blot of CF patients' sera colonized by Scedosporium against S. boydii $24 \mathrm{~h}$ total protein extract (B). Decreasing acrylamide \% to 6 (C, red square) allowed to better visualize high Mr antigens (D).

The main reactivity was lost after membrane oxidation with $50 \mathrm{mM}$ sodium metaperiodate. This treatment allowed to visualize strictly protein antigens recognized by the pooled sera from the three groups of CF patients.

Sera from CF patients with Scedosporium recognized 3 protein antigens with 60-62 kDa and 4.2-4.4 pl (red arrows), which were not detected by sera from the other CF groups (E-G).

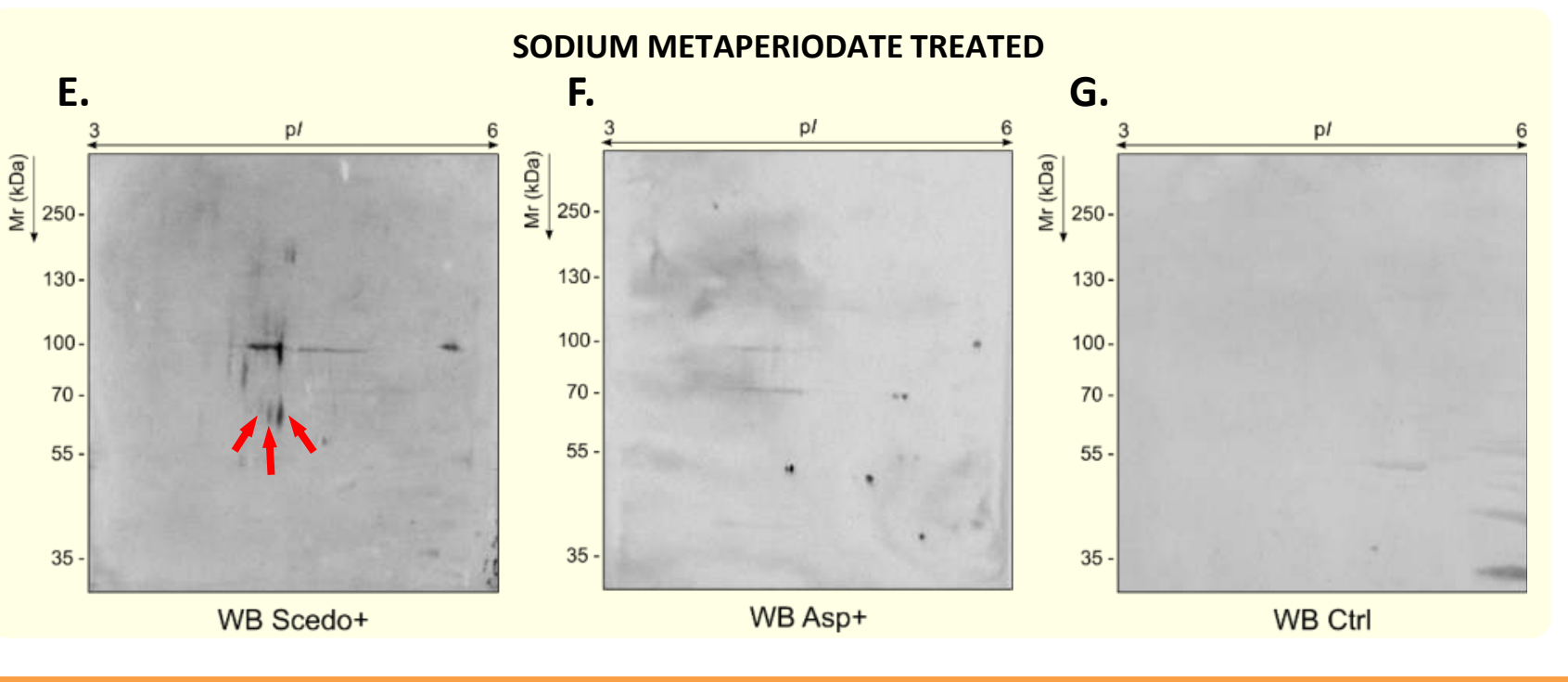

Social networks

https://www.facebook.com/microbiomicsEHU/ https://twitter.com/microbiomicsehu https://www.instagram.com/microbiomics/
Acknowledgements

$\checkmark$ This work has been supported by grants (GIU15/36 and PPG17/41) from the UPV/EHU. $\checkmark$ LM-S, IB and AA were supported by predoctoral fellowships from the Basque Government.

\section{CONCLUSION}

CF patients colonized by Scedosporium recognize specifically 3 antigens from $S$. boydii with 60$62 \mathrm{kDa}$ and 4.2-4.4 pl, which will be studied as diagnostic targets to develop a sensitive and specific serological test. 